\title{
A rapid and efficient autoclave pre-treatment to extract iodine-129 from urine samples for AMS analysis
}

\author{
Fahad A. AL-Otaibi, Matthew N. Herod, R. Jack Cornett
}

\begin{abstract}
A new method was developed to extract ${ }^{129}$ I from urine samples and then measure it by AMS. The samples were pre-treated in an autoclave with hydrogen peroxide to remove unwanted compounds from the urine samples and were acidified with nitric acid, followed by precipitation of iodine as silver iodide (AgI) for measurement by AMS. This new procedure is substantially faster than previous methods for the extraction of iodine from urine and results in less chemical waste. The efficiency and reproducibility of this method were evaluated by using ${ }^{125} \mathrm{I}$ as a yield tracer, eventually giving a recovery above $99 \%$. To achieve this, several iterations of the method were required. The method was then successfully applied to measure ${ }^{129} \mathrm{I} /{ }^{127} \mathrm{I}$ isotopic ratios and ${ }^{129} \mathrm{I}$ concentrations in 25 human urine samples. The AMS results for ${ }^{129} \mathrm{I}$ in urine ranged $3.3 \times 10^{6}$ atoms/L to $884 \times 10^{6}$ atoms/L and the isotope ratio $\left({ }^{129} \mathrm{I} /{ }^{127} \mathrm{I}\right)$ in human urine ranged from $7.38 \times 10^{-12}$ to $3.97 \mathrm{x}$ $10^{-10}$ with a median of $1.29 \times 10^{-10}$. This new method will be useful for investigations into the sources of iodine in the human diet and their relative importance for iodine sufficiency.
\end{abstract}




\section{Introduction:}

${ }^{129} \mathrm{I}$ is a long-lived radioisotope with a half-life of 16.14 million years (García-Toraño et al. 2018). It is produced naturally by the nuclear interaction of cosmic rays with xenon in the upper atmosphere and by the spontaneous fission of uranium-238 in minerals (Raisbeck and Yiou 1999). Since 1945, the concentration of ${ }^{129} \mathrm{I}$ in the environment has increased sharply due to anthropogenic nuclear activities such as nuclear weapons testing, nuclear fuel reprocessing plants (NFRP), and a minor contribution from nuclear power plant (NPP) accidents and emissions. A study by Zhang et al. 2013 differentiated ${ }^{129} \mathrm{I} /{ }^{127} \mathrm{I}$ ratios into three categories: pre-nuclear era natural levels with ${ }^{129} \mathrm{I} /{ }^{127} \mathrm{I}$ ratios of $10^{-12}$, modern background ${ }^{129} \mathrm{I} /{ }^{127} \mathrm{I}$ ratios ranging from $10^{-11}$ to $10^{-9}$, and contaminated areas that have ${ }^{129} \mathrm{I} /{ }^{127} \mathrm{I}$ ratios from $10^{-8}$ higher. As a mobile long-lived radioisotope in the hydrosphere and atmosphere, iodine is distributed throughout the environment and can easily enter the food chain through the hydrosphere, atmosphere, and geosphere.(Zhang and Hou 2013; Jabbar, Wallner, and Steier 2013). Since ${ }^{129} \mathrm{I}$ is a fission product, it is a very good indicator of the global dispersion of nuclear emissions, without being itself a dangerous radioisotope.

High ${ }^{129} \mathrm{I}$ concentrations can be measured by activity counting methods such as liquid scintillation counting (LSC), $\gamma$-spectrometry, or neutron activation analysis (NAA) as well as mass spectrometry techniques such as Inductively Coupled Plasma-Mass Spectrometry (ICP-MS) and Accelerator Mass Spectrometry (AMS). Of these methods, AMS is the only one that can measure

${ }^{129} \mathrm{I}$ in environmental and biological samples at the low abundances typically found outside of contaminated areas.

Applications of ${ }^{129}$ I are typically limited to environmental investigations such as its use as a tracer in oceanography, groundwater dating or of nuclear emissions. Due to the sensitivity of AMS, there is an opportunity to expand the use of ${ }^{129}$ I applications into new fields such as biomedical 
applications or nutrition, which have been investigated less frequently. One hurdle to expanding ${ }^{129} \mathrm{I}$ applications is extracting ${ }^{129} \mathrm{I}$ from biological samples for AMS analysis. Due to its low concentration in the environment, ${ }^{129} \mathrm{I}$ analysis requires chemical separation that must consider many factors such as sample type, sample size, and iodine chemical species (Zhao et al. 2009; Zhang and Hou 2013; Jabbar, Wallner, and Steier 2013). ${ }^{129}$ I pre-treatment procedures such as UV-irradiation, caustic fusion, acid decomposition, oxidant decomposition, alkaline leaching, ashing, combustion, and microwave digestion have all been used with varying degrees of complexity and success, to prepare ${ }^{129}$ I samples for AMS analysis (Herod et al. 2014; GómezGuzmán et al. 2011; Muramatsu et al. 2008; Zhao et al. 2009; Zhang and Hou 2013).

Regarding ${ }^{129}$ I in urine, there is only one study by (Hou et al. 2003) that measured ${ }^{129}$ I in three urine samples, using neutron activation analysis (NAA). (Hou et al. 2003) used three to four liters of urine and compared ${ }^{129} \mathrm{I}$ in animal thyroid and human urine. To date, no studies have measured ${ }^{129} \mathrm{I}$ in urine by accelerator mass spectrometry.

Urine is a human waste product that is typically excreted in a range of $600 \mathrm{ml}$ to $2000 \mathrm{ml}$ daily and is a common clinical sample. In general, $95 \%$ of urine is water, and $5 \%$ is dissolved solutes (Strasinger.2008). Urine contains a high concentration of urea, organic substances such as creatinine, and inorganic elements, which are primarily chloride followed by sodium, potassium, and trace amounts of others (Strasinger.2008).

This paper aims to demonstrate a reliable and reproducible method for the measurement of ${ }^{129} \mathrm{I}$ in human urine, by using thermal-pressure digestion for AMS and by relating the result to iodine sources in the diet in a preliminary sense. The initial results from the method are presented and compared to those of (Hou et al. 2003). The efficiency and reproducibility of this method were evaluated using ${ }^{125} \mathrm{I}$ as a tracer of recovery, and a discussion on the factors that affect the 
extraction efficiency and AMS measurements is presented. This study is a first step towards understanding the sources of ${ }^{127} \mathrm{I}$ and ${ }^{129} \mathrm{I}$ in the human diet using ${ }^{129} \mathrm{I}$ as a nutritional tracer. This procedure is envisaged to potentially expand the applications of ${ }^{129}$ I into non-environmental fields.

\section{Methods:}

\subsection{Chemicals and Instruments}

Urine samples were digested in $60 \mathrm{ml}$ borosilicate glass tubes in an autoclave (Tuttnauer Brinkmann). ${ }^{125}$ I tracer solution (37 MBq) was ordered from PerkinElmer and diluted in $14.9 \mathrm{M}$ $\mathrm{NH}_{4} \mathrm{OH}$ to give an activity of $20,000 \mathrm{~Bq} / \mathrm{ml}$ for use as a recovery tracer. All solutions were prepared using $18 \mathrm{M} \Omega$ de-ionized water. The parameters used for autoclave digestion are given in Table 1.

A PerkinElmer Wizard 2 Automatic Gamma Counter (2470-0020) with two well type $\mathrm{NaI}$ detectors was used to measure ${ }^{125}$ I to quantify the extraction efficiency. A 3MV HVE tandem AMS system was used to measure the concentrations of ${ }^{129} \mathrm{I}$ in the AgI extracted from the urine at the Andre E. Lalonde Accelerator Mass Spectrometry Laboratory. The technical details of the ${ }^{129}$ I measurement by AMS are described in (Francisco et al. 2020) and ion source parameters are given in Table 2. An Agilent 8800 ICP-MS triple quadrupole was used to measure the concentrations of ${ }^{127} \mathrm{I}$.

Table 1: Autoclave parameters

\begin{tabular}{ll} 
Parameter & Value \\
\hline Program & Wrapped Instruments and Porous Loads \\
Temperature & $250^{\circ} \mathrm{F}\left(121^{\circ} \mathrm{C}\right)$ \\
Digestion Time & $20 \mathrm{~min}$ \\
Dry time & $60 \mathrm{~min}$
\end{tabular}


Table 2: AMS ion source parameters (adapted from Francisco et al. 2020)

\begin{tabular}{ll} 
Parameter & Value \\
\hline Cesium reservoir temperature & $80{ }^{\circ} \mathrm{C}$ \\
Target Voltage & $7.0 \mathrm{kV}$ \\
Target current & $0.5-1.5 \mathrm{~mA}$ \\
Extraction Voltage & $28.0 \mathrm{kV}$ \\
Extraction current & $0.2 \mathrm{~mA}$ \\
Ionizer current & $18 \mathrm{~A}$ \\
Vacuum (mbar) & $0.5-1.0 \mathrm{mBar}$
\end{tabular}

\section{$2.2{ }^{127}$ I analysis by inductively coupled plasma mass spectrometry.}

The most commonly used method to measure iodine in urine is direct dilution and ICP-MS analysis. In this procedure, we considered FDA guidelines for method validation (FDA 2018), and we applied a modified procedure of (Allen et al.1990) as follows: $1 \mathrm{~g}$ of urine sample was mixed with 9 grams of $1 \% 0.292 \mathrm{M}$ ammonium hydroxide $\left(\mathrm{NH}_{4} \mathrm{OH}\right)$ to obtain a 1/10 dilution. To avoid any sample memory during the analysis, the samples were diluted and additional 50 times. Indium-115 and tellurium-205 were used as internal standards to obtain optimal precision and to determine the accuracy of the ICP-MS instrument. Seven standards (0ppb), (1.56ppb), (3.125ppb), (6.25ppb), (12.5ppb), (25ppb), and (50ppb) were used to develop a calibration curve to determine ${ }^{127} \mathrm{I}$ in urine. The limit of detection was $0.0445 \mathrm{ppb}$, and the limit of quantification was $0.148 \mathrm{ppb}$.

\subsection{Sample collection and preparation}

Human urine samples were collected in sterile, dry, $200 \mathrm{ml}$ polypropylene, disposable containers from 27 individuals living in Ottawa, Ontario, Canada. Their ages ranged from 3 years old to more than 66 years old (Table 3). Ethics approval was obtained from the University of Ottawa, and participants were provided with consent forms and surveys about their eating 
habits (Figure 1). The participants were advised to fill the container in the early morning as morning urine typically has a higher specific gravity, i.e., less water and more solute than urine from other times of day (Strasinger 2008). The minimum urine volume required to perform the experiments was $100 \mathrm{ml}$.

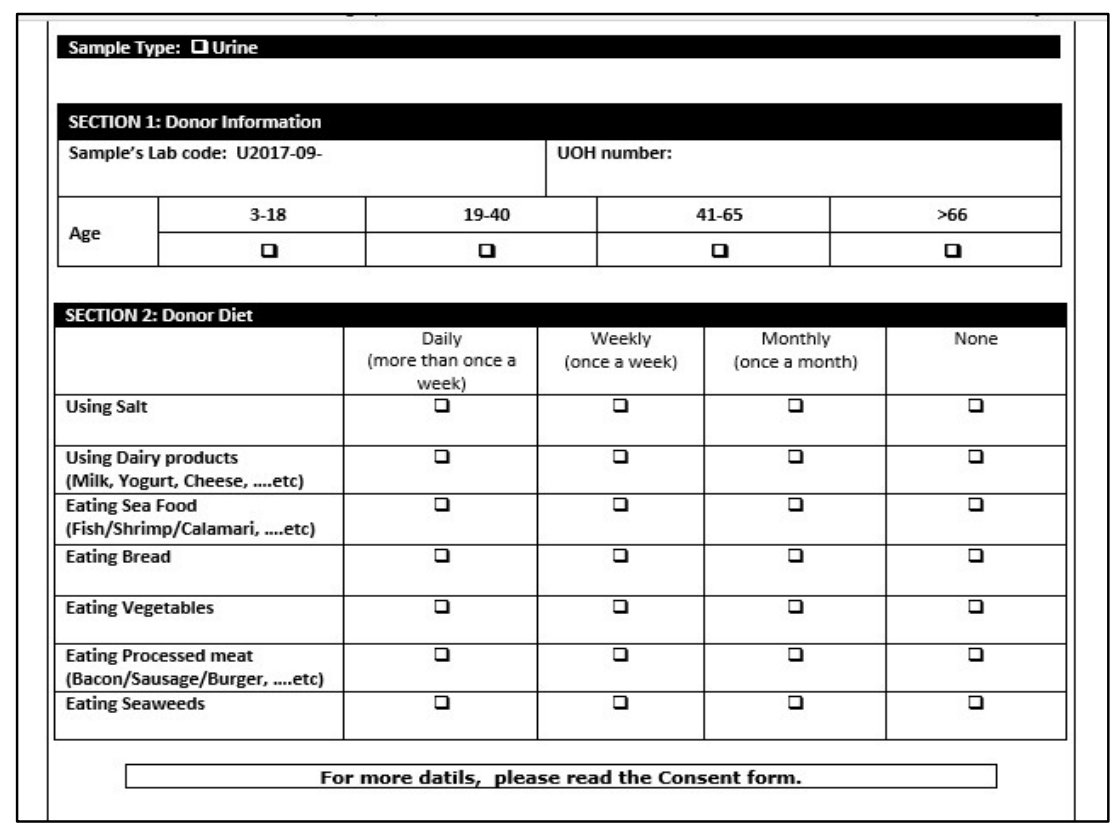

Figure 1: An example of the questionnaire given to all participants

\begin{tabular}{lr}
\multicolumn{1}{c}{ Age (years) } & Number \\
\hline 3 to 18 & 4 \\
19 to 40 & 14 \\
41 to 65 & 8 \\
$>66$ & 1
\end{tabular}

Total number $\quad 27$

$\underline{\text { Table } 3 \text { Participants demographic information }}$ 


\subsection{Extraction of ${ }^{129}$ I from urine for AMS analysis}

Four progressive iterations were required to develop the optimal procedure to extract ${ }^{129} \mathrm{I}$ from the urine samples. In order to improve the yield of iodine and to minimize the chemical waste, the fourth iteration that uses less $\mathrm{HNO}_{3}$ and less $\mathrm{NH}_{4} \mathrm{OH}$ was applied to all samples for the extraction to analyse the samples by AMS.

In the fourth, and final, extraction procedure, $25 \mathrm{~g}$ of urine plus $5 \mathrm{~g}$ of hydrogen peroxide were treated in the autoclave. After digestion in the autoclave, twenty milligrams of sodium carbonate $\mathrm{Na}_{2} \mathrm{CO}_{3}$ was added and heated under hot plate for $10 \mathrm{~min}$ to remove any residual hydrogen peroxide. The samples were then acidified using $0.2 \mathrm{ml}$ of $15.66 \mathrm{M} \mathrm{HNO}_{3}$ to lower the $\mathrm{pH}$ to around one, and two milligrams of iodine as NaI carrier with a ${ }^{129} \mathrm{I} /{ }^{127} \mathrm{I}$ ratio of $1.4 \times 10^{-14}$ was added. To precipitate a sample of AgI for AMS, $200 \mu \mathrm{l}$ of $0.1 \mathrm{M} \mathrm{AgNO}_{3}$ was added. The samples were left for 12 hours in darkness to allow the precipitate to settle. The precipitate was collected and washed in de-ionized water and $1 \mathrm{ml}$ of $14.99 \mathrm{M}$ ammonium hydroxide $\mathrm{NH}_{4} \mathrm{OH}$ was added to dissolve any co- precipitating $\mathrm{AgCl}$. The samples were centrifuged and the supernatant removed. The precipitate was washed in de-ionized water and centrifuged. The samples were dried in the oven at $60^{\circ} \mathrm{C}$ for overnight. The AgI precipitate was then prepared for AMS analysis by mixing it with pure niobium powder in a ratio of $1: 1$ or $1: 2$ by volume and pressing the mixture into a copper cathode to make the AMS target. A flow chart of the method is shown in Fig 2. 


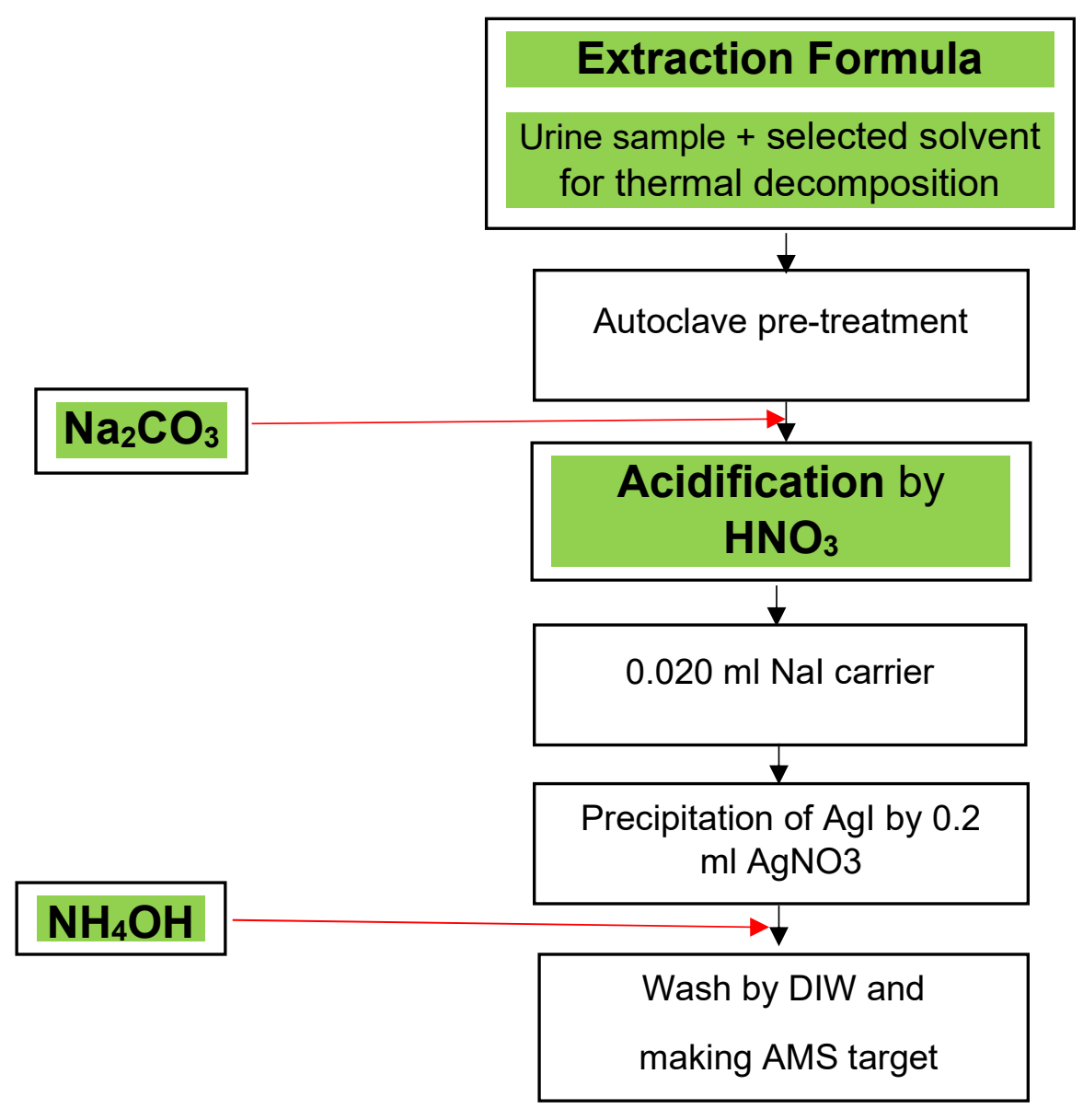

Figure2. Flow chart showing the chemical extraction of ${ }^{129}$ I in the urine. Steps in the bold were varied among the iterations.

\subsection{Blank}

Several blanks were used during this analysis. Deionized water was used as a processed blank during the digestion and extraction chemistry procedure, giving isotopic ratio. In AMS run, sodium iodide (NaI) blank carrier, and niobium powder blank were used and their total counts and ratio were constant every ten samples. In ICP-MS run, $1 \% \mathrm{NH}_{4} \mathrm{OH}$ was used as blank and its concentration was constant every ten samples too. This indicates that no sample cross contamination occurs during the run in both instruments. 


\section{Results and Discussion}

\subsection{Experimental optimization}

\subsubsection{Optimization of iodine extraction from urine}

Biological samples, such as urine, contain variously abundant components and elements, generally termed matrix, which often affect extraction recovery and reproducibility for rare isotopes. In order to measure ${ }^{129} \mathrm{I}$ in urine samples by AMS, the sample preparation should recover sufficient iodine through reduction of these matrix effects. In the case of iodine in urine, sample matrix is usually eliminated either by acid digestion or alkaline ashing (May et al. 1997). In this method, autoclave pre-treatment is used as an alternative to the conventional extraction techniques for ${ }^{129} \mathrm{I}$, which typically use strong acids or bases to digest the sample (May et al. 1997; Zhao et al. 2009). For the digestion, autoclave and microwave provide the same outcome of thermal breakdown (Taylor et al. 1996). However, the autoclave is generally more affordable than lab microwaves. The ability of the autoclave to break down organic matter is well known and it can be used to digest organic matter and compounds such as urine, if it is used with a proper digestion solvent (Jeffries, Dieken, and Jones 1979; Crowther 1978; Navarrete-López et al. 2012). Using an autoclave to extract iodine from samples is uncommon in the literature (Haldimann, Eastgate, and Zimmerli 2000); therefore, the evaluation of this technique with respect to iodine recovery was necessary to ensure all iodine remained in the sample. The recovery of iodine during autoclave pre-treatment was tested using the addition of a known amount of ${ }^{125}$ I to determine the efficiency of the procedure.

\subsubsection{Chloride interference}


In the first and second iterations, potassium persulfate was the digestion solvent used. For the first iteration, $0.4 \mathrm{~g}$ of urine was mixed with $1 \mathrm{~g}$ of potassium persulfate then dissolved in 20 $\mathrm{g}$ of de-ionized water and digested in the autoclave. The preliminary AMS results showed that ${ }^{129}$ I concentration was low and too close to the AMS background to obtain reliable data. Therefore, a second iteration with increased sample volume was attempted to increase the AMS signal. A $4 \mathrm{~g}$ urine sample was mixed with $1 \mathrm{~g}$ potassium persulfate then dissolved in $200 \mathrm{~g}$ of de-ionized water and digested. The sample was precipitated using the method described above; however, the resulting precipitate was initially white and became gray over time. A portion of this gray precipitate was collected and analyzed using a scanning electron microscope which showed a significant amount of chlorine relative iodine suggesting that $\mathrm{AgCl}$ was coprecipitating with AgI (Table 4). This resulted in poor currents in the AMS ion source (Brunzel 2013).

$\begin{array}{ccccccccc}\text { Sample 1 } & \mathrm{Al} & \mathrm{Cl} & \mathrm{Ag} & \mathrm{I} & \mathrm{O} & \mathrm{K} & \mathrm{S} & \text { Total \% } \\ \text { Spectrum 1 } & 0.48 & \mathbf{2 2 . 7 1} & 66.01 & \mathbf{5 . 4 8} & 5.32 & & 100 \\ \text { Spectrum 2 } & 0.00 & \mathbf{2 2 . 4 5} & 67.20 & \mathbf{5 . 3 7} & 4.98 & 100 \\ \text { Spectrum 3 } & 0.00 & \mathbf{1 6 . 3 0} & 72.06 & \mathbf{6 . 2 9} & 5.34 & 100 \\ \text { Spectrum 4 } & 19.27 & \mathbf{1 7 . 6 2} & 42.79 & \mathbf{0 . 0 0} & 20.31 & 100\end{array}$

$\begin{array}{llllllll}\text { Sample 2 } & & & & & & & \\ \text { Spectrum 1 } & \mathbf{1 8 . 7 4} & 59.78 & \mathbf{3 . 2 2} & 10.53 & 4.23 & 3.49 & 100 \\ \text { Spectrum 2 } & \mathbf{1 8 . 7 4} & 63.80 & \mathbf{3 . 2 3} & 9.17 & 2.43 & 2.63 & 100\end{array}$

Table 4: Scanning Electron Microscope results show chloride interferences for two samples from iteration two. Units in \%

The results of these initial experiments raise a number of questions regarding the efficiency of digestion solvent, the required sample volume and how to eliminate interference from coprecipitation of $\mathrm{AgCl}$. 


\subsubsection{Iodine loss due to volatilization}

To resolve the problems identified in the first two iterations, potassium persulfate was replaced with hydrogen peroxide as the solvent, and the ratio of sample volume to solvent was changed to $5 \mathrm{ml}$ urine $+0.5 \mathrm{ml} \mathrm{H}_{2} \mathrm{O}_{2}$ (10:1) in order to reduce dilution. $20 \mathrm{mg}$ sodium carbonate, $\mathrm{Na}_{2} \mathrm{CO}_{3}$, was added after the autoclave digestion to remove residual hydrogen peroxide (Wu and Englehardt 2012). $3 \mathrm{~mL}$ of ammonium hydroxide was added at intervals before drying to eliminate chloride. A portion of the precipitate was collected and analyzed using a scanning electron microscope which showed a large reduction in the chloride interference (Table 5). However, both gamma spectrometry of the ${ }^{125}$ I tracer and AMS of ${ }^{129}$ I results showed that there was poor recovery despite these changes. We hypothesize that the loss of iodine from samples was related to the addition of nitric acid after the autoclave pre-treatment, which oxidized the iodine into the volatile $I_{2}$ species. Therefore, the acid volume was decreased in the fourth iteration from 1 milliliter to 0.2 millilitres to reduce iodine volatilization.

\begin{tabular}{cccccccc}
\hline Sample & $\mathrm{Al}$ & $\mathrm{Cl}$ & $\mathrm{Ag}$ & $\mathrm{I}$ & $\mathrm{O}$ & $\mathrm{C}$ & Total \% \\
Spectrum 1 & 0.00 & 0.00 & 39.29 & $\mathbf{4 5 . 0 3}$ & 2.62 & 13.06 & 100 \\
Spectrum 2 & 0.00 & 0.00 & 38.85 & $\mathbf{4 4 . 3 5}$ & 3.11 & 13.69 & 100
\end{tabular}

Table 5: SEM results show the absence of chloride interferences in the precipitate. Units are \%..

\subsubsection{Effect of autoclave digestion and solvent on ${ }^{125}$ I recovery}

In order to determine if there was any loss of iodine in the autoclave, ${ }^{125}$ I was added as a yield tracer. Using ${ }^{125} \mathrm{I}$ as a quantitative recovery tracer for ${ }^{127} \mathrm{I}$ and ${ }^{129} \mathrm{I}$ has been reported in detail elsewhere (Herod et al. 2014). Briefly, the method uses the coincidence sum gamma counting method to determine absolute sample activity (Horrocks and Klein 1974). The use of

${ }^{125}$ I as a quantitative tracer of iodine recovery enabled us to determine if digesting the samples in 
the autoclave led to a loss of iodine. The data in Table 6 confirm that heat and pressure in autoclave digestion has no effect on ${ }^{125} \mathrm{I}$ recovery and, therefore, is not expected to cause a loss of ${ }^{129} \mathrm{I}$ or ${ }^{127} \mathrm{I}$ from the samples. In the first iteration, potassium persulfate was used as a digestion solvent because the persulfate digestion is known as a safe oxidizing agent used in iodine analysis (Pino, Fang, and Braverman 1996) but it requires more water and produces substantial chemical waste. Thus, another safe oxidizing agent such as hydrogen peroxide was used as a digestive solvent along with an increase in the amount of urine sample. The results of the ${ }^{125} \mathrm{I}$ yield tracer for each solvent after digestion in the autoclave and show that the recovery obtained from $\mathrm{H}_{2} \mathrm{O}_{2}$ was superior to that of $\mathrm{K}_{2} \mathrm{~S}_{2} \mathrm{O}_{8}$. As a result, $\mathrm{H}_{2} \mathrm{O}_{2}$ was chosen as the solvent for the optimized procedure as it is both more efficient and produced less waste.

$\begin{array}{lcc} & \text { Total Activity }(\mathbf{B q}) & \text { Recovery \% to Pure }{ }^{\mathbf{1 2 5}} \mathbf{I} \\ \text { Pure }{ }^{125} \mathrm{I} & 2372.9 & 100 \\ \text { Blank(air) } & 0 & 0 \\ \text { Mean value of }{ }^{125} \mathrm{I} \text { standards }(\mathrm{n}=4) & 2381 & 100.3 \\ \text { Mean value of }{ }^{125} \mathrm{I} \text { in urine with } \mathrm{K}_{2} \mathrm{~S}_{2} \mathrm{O}_{8}(\mathrm{n}=3) & 2157.4 & 90 \\ \text { Mean value of }{ }^{125} \mathrm{I} \text { in urine with } \mathrm{H}_{2} \mathrm{O}_{2}(\mathrm{n}=3) & 2509.9 & 105 \\ & & \text { Recovery \% to standard } \\ \text { Mean value of }{ }^{125} \mathrm{I} \text { in urine with } \mathrm{K}_{2} \mathrm{~S}_{2} \mathrm{O}_{8}(\mathrm{n}=3) & 2157.4 & 90.6 \\ \text { Mean value of }{ }^{125} \mathrm{I} \text { in urine with } \mathrm{H}_{2} \mathrm{O}_{2}(\mathrm{n}=3) & 2509.9 & 105\end{array}$

Table.6: Analytical results of ${ }^{125}$ I yield for the two-solvents $(n=6)$ used in the extractions after autoclave digestion compared to mean values of ${ }^{125} \mathrm{I}$ in water $(\mathrm{n}=4)$ without autoclave digestion.

\subsubsection{Comparison of ${ }^{125}$ I recovery for each iteration}

The ${ }^{125}$ I recovery for each iteration were compared and the results are summarized in Table 7. The second iteration was not tested as it was identical to the first except for an increased volume of the sample and volume of solvent. 


\begin{tabular}{|c|c|c|c|}
\hline Iteration & Total activity $(\mathrm{Bq})$ & Recovery (\%) & $\begin{array}{c}\text { \% Recovery } \\
\text { Error }\end{array}$ \\
\hline \multicolumn{4}{|l|}{ First } \\
\hline & 8765.6 & 42.4 & 0.00020 \\
\hline & 8206.8 & 39.7 & 0.00021 \\
\hline & 8351.5 & 40.4 & 0.00021 \\
\hline \multicolumn{4}{|l|}{ Third } \\
\hline & 584.6 & 2.8 & 0.00069 \\
\hline & 536.8 & 2.6 & 0.00073 \\
\hline & 555.2 & 2.7 & 0.00071 \\
\hline \multicolumn{4}{|l|}{ Fourth } \\
\hline & 20739.9 & 100.4 & 0.00013 \\
\hline & 20718.1 & 100.3 & 0.00013 \\
\hline & 20667.2 & 100 & 0.00014 \\
\hline
\end{tabular}

Table.7: A comparison table of the iterations in terms of ${ }^{125}$ I recovery shows that the fourth extraction procedure has more sensitivity and reproducibility $(\mathrm{n}=9)$.

The first iteration had a poor recovery of ${ }^{125}$ I of less than $50 \%$ due to the low sample volume and interference from chloride in the samples, while the third experiment had very poor recovery of $\sim 3 \%$ due to volatilization of iodine from the sample during acidification. The loss of iodine from samples in the first and third iteration is not related to autoclave heat and pressure as the results in Table 6 showed no loss of ${ }^{125} \mathrm{I}$ following the autoclave digestion step. The fourth iteration decreased the amount of acid used and as a result an excellent recovery of ${ }^{125}$ I, exceeding $99 \%$, was obtained.

\section{$3.2^{129} I$ and ${ }^{127}$ I concentrations in urine}

The fourth iteration of the method was successfully applied to measure ${ }^{129} \mathrm{I}$ in urine samples from 25 people. The AMS results for ${ }^{129} \mathrm{I}$ in urine ranged from $3.3 \times 10^{6}$ atoms $/ \mathrm{L}$ to $884 \times 10^{6}$ atoms $/ \mathrm{L}$ with a median of $108.7 \times 10^{6}$ atoms $/ \mathrm{L}$. The results of ${ }^{127} \mathrm{I}$ in the urine sample ranged from $24.1 \mu \mathrm{g} / \mathrm{L}$ to $3544 \mu \mathrm{g} / \mathrm{L}$ with a median of $160.4 \mu \mathrm{g} / \mathrm{L}$. The ${ }^{129} \mathrm{I} /{ }^{127} \mathrm{I}$ ratio ranged from $7.38 \mathrm{x}$ 
$10^{-12}$ to $3.97 \times 10^{-10}$ with a mean of $1.36 \times 10^{-10}$ and a median of $1.29 \times 10^{-10}$. All results are summarized in Table 8.

\begin{tabular}{|c|c|c|c|c|}
\hline Sample ID & $\begin{array}{c}{ }^{127} \mathrm{I} \text { concentration } \\
\mu \mathrm{g} / \mathrm{L} \\
\text { with standard error } \\
\pm 0.24\end{array}$ & $\begin{array}{c}\text { Corrected } \\
{ }^{129} \mathrm{I} \text { Concentration } \\
10^{6} \mathrm{~atm} / \mathrm{L}\end{array}$ & $\begin{array}{c}\text { Error of } \\
{ }^{129} \text { I Concentration } \\
10^{6} \mathrm{~atm} / \mathrm{L}\end{array}$ & $\begin{array}{c}{ }^{129} \mathrm{I} /{ }^{127} \mathrm{I} \text { ratio } \\
10^{-10}\end{array}$ \\
\hline 1 & 225.6 & 238.3 & 6.65 & 2.23 \\
\hline 2 & 79.7 & 61.8 & 2.74 & 1.64 \\
\hline 3 & 3543.9 & 884.0 & 20.22 & 0.56 \\
\hline 4 & 85.0 & 109.5 & 2.56 & 2.72 \\
\hline 5 & 94.4 & 3.3 & 2.33 & 0.07 \\
\hline 6 & 1197.0 & 798.6 & 18.31 & 1.41 \\
\hline 7 & 207.2 & 217.3 & 4.63 & 2.21 \\
\hline 8 & 339.7 & 318.4 & 7.51 & 1.98 \\
\hline 9 & 94.8 & 16.1 & 1.01 & 0.36 \\
\hline 10 & 255.4 & 40.2 & 1.76 & 0.33 \\
\hline 11 & 24.1 & 15.3 & 1.28 & 1.33 \\
\hline 12 & 143.1 & 87.7 & 2.49 & 1.29 \\
\hline 13 & 139.6 & 180.2 & 3.61 & 2.72 \\
\hline 14 & 482.5 & 84.5 & 2.63 & 0.37 \\
\hline 15 & 394.8 & 340.6 & 17.05 & 1.82 \\
\hline 16 & 185.9 & 264.6 & 5.65 & 3.00 \\
\hline 17 & 338.8 & 108.7 & 2.66 & 0.68 \\
\hline 18 & 122.6 & 52.2 & 2.61 & 0.90 \\
\hline 19 & 81.0 & 12.4 & 3.36 & 0.32 \\
\hline 20 & 160.4 & 50.8 & 2.78 & 0.67 \\
\hline 21 & 131.0 & 32.1 & 2.23 & 0.52 \\
\hline 22 & 83.3 & 156.6 & 3.25 & 3.97 \\
\hline 23 & 102.3 & 14.2 & 0.87 & 0.29 \\
\hline 24 & 441.8 & 231.0 & 8.21 & 1.10 \\
\hline 25 & 222.7 & 157.5 & 4.40 & 1.49 \\
\hline
\end{tabular}

Table 8: Concentration of ${ }^{129}$ I and ${ }^{127} I$ in human urine from ICP-MS and AMS ( $\mathrm{n}=25$ )

Urinary iodine is highly variable from day to day (Zimmermann 2008). The results in Table 8 show the concentration of ${ }^{127} \mathrm{I}$ on the day of collection. Figure 3 shows the frequency distribution of ${ }^{127} \mathrm{I}$ in the samples. One sample had a very low ${ }^{127} \mathrm{I}$ concentration of $24.1 \mu \mathrm{g} / \mathrm{L}$ which could be related to the age of that particular participant, who was 3 years old. Six samples were 
within mild deficiency concentration range (50-99 $\mu \mathrm{g} / \mathrm{L})$, eleven samples were within the optimal concentration range (100-299 $\mu \mathrm{g} / \mathrm{L})$, and five samples were within the risk of hyperthyroidism concentration range (300-499 $\mu \mathrm{g} / \mathrm{L})$. There were two samples which significantly exceeded the high concentration range $\left({ }^{127} \mathrm{I}\right.$ above $\left.500 \mu \mathrm{g} / \mathrm{L}\right)$ and exceeded $1000 \mu \mathrm{g} / \mathrm{L}$. It is likely these extremely high values are due to thyroid diseases or iodine supplements taken by those participants. The variables ${ }^{127} \mathrm{I}$ and ${ }^{129}$ I were found to be positively correlated when these high samples were included, with a Pearson $r=0.84(n=25)$. If we consider the ${ }^{127}$ I values above 500 $\mu \mathrm{g} / \mathrm{L}$ as outliers and eliminate those samples from the Pearson correlation analysis, the variables ${ }^{127} \mathrm{I}$ and ${ }^{129} \mathrm{I}$ were still found to be significantly correlated with a Pearson $\mathrm{r}=0.56(\mathrm{n}=23)$. In both scenarios, the ${ }^{129}$ I concentration had a statistically significant correlation with the ${ }^{127} I$ concentration (Fig 4). This positive correlation suggests that may be a common source of ${ }^{129} \mathrm{I}$ and ${ }^{127} \mathrm{I}$ in the human body. In environmental samples, including those from other studies analysing ${ }^{129} \mathrm{I}$ in North America, ${ }^{129} \mathrm{I}$ and ${ }^{127} \mathrm{I}$ are rarely well correlated due to their differing sources (Herod et al. 2016; Fehn 1999). This is the first time such a correlation has been measured in humans and suggests that ${ }^{129} \mathrm{I}$ could be a potential tracer of dietary ${ }^{127} \mathrm{I}$ as it appears the integration of these two iodine isotopes is occurring in nature prior to their consumption by humans. Nutritional literature generally presumes that most ${ }^{127} \mathrm{I}$ in the human body is from iodized salt (Pandav et al. 2013; Fields and Borak 2009; Gunnarsdottir and Dahl 2012) and salt iodination programs have been extremely successful in reducing hypothyroidism due to lack of sufficient dietary iodine, particularly in developing nations (Mannar and Dunn 1995; de Benoist et al. 2004). However, these results could indicate that the source of iodine in humans is mixed as opposed to being solely from iodized salt and that whatever the principal sources of iodine to humans is, they include ${ }^{129} \mathrm{I}$ as well. It is clear from this data that additional dietary sources of 
iodine may be important contributors of both ${ }^{129} \mathrm{I}$ and ${ }^{127} \mathrm{I}$ and their importance as iodine sources should be the subject of further study.

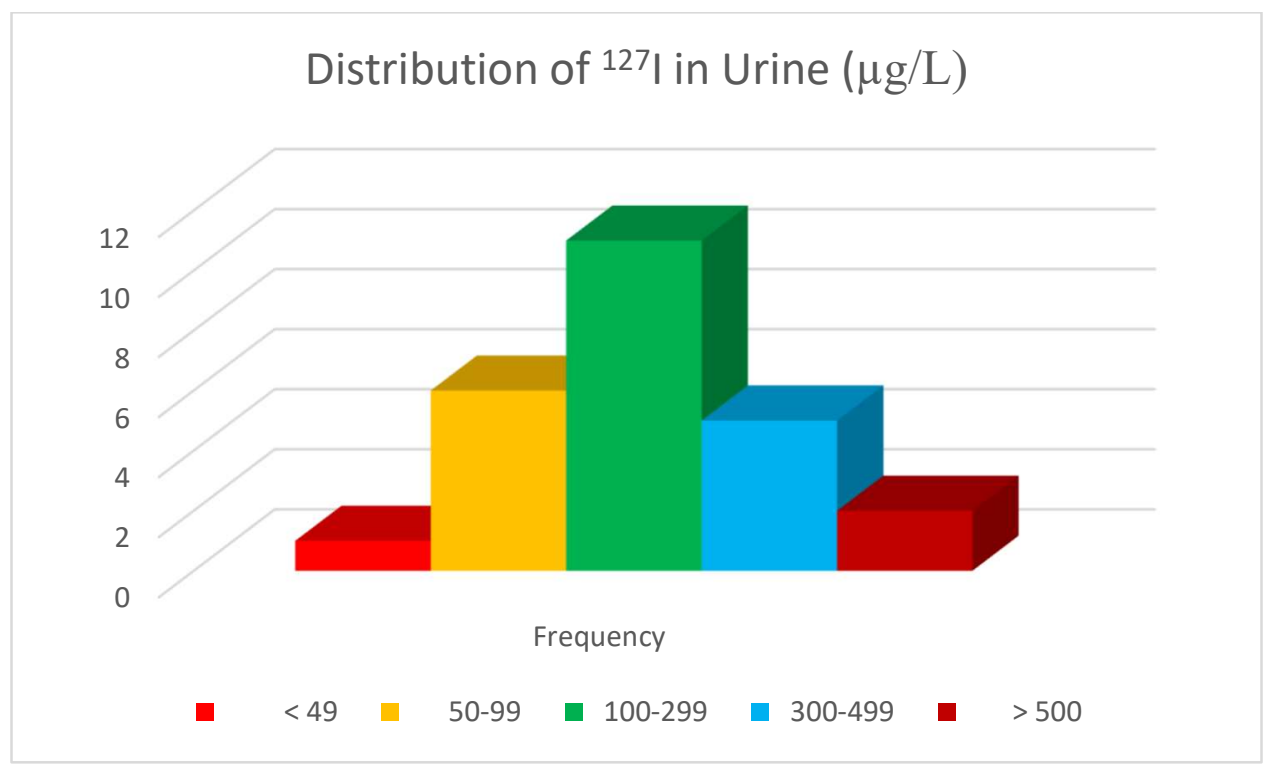

Figure 3: Frequency distribution of ${ }^{\mathbf{1 2 7}} \mathbf{I}$ concentration data based on $\mathbf{5}$ categories. Less than 49 is mild deficiency, 50-99 is moderate deficiency, 100-299 is optimal concentration, 300-499 is risk of hyperthyroidism concentration, and $>500$ is high concentration.

\section{${ }^{129}$ vs $^{127}$ I in Urine}

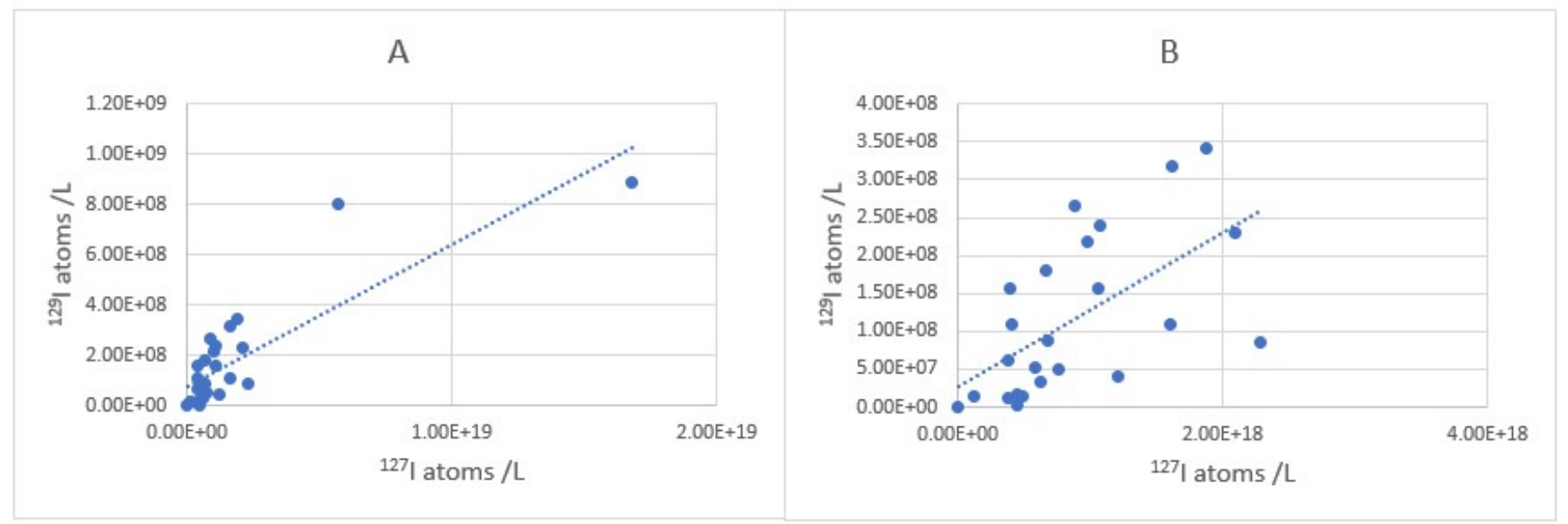


Figure 4: Linear regressions showing correlation of ${ }^{129} \mathrm{I}$ concentration expressed as atoms/L with ${ }^{127} \mathrm{I}$ atoms/L (A) $R^{2}=0.701, p<0.01, n=25,(B) R^{2}=0.32, p<0.01, n=23$.

The main sources of ${ }^{129}$ in the environment are from human nuclear activities specifically, nuclear fuel reprocessing plants in Europe and to a lesser degree, Asia. The release of ${ }^{129} \mathrm{I}$ from all sources, dominated by fuel reprocessing, is estimated at $5.21 \times 10^{11} \mathrm{~Bq} / \mathrm{year}$, giving an estimated dose equal to $3.36 \times 10^{-6} \mathrm{mSv} / \mathrm{year}$ (Sunny et al. 2014). Although there is no radiation risk for human health at this current environmental dose level, the accumulation of ${ }^{129} \mathrm{I}$ in the human body should be investigated as it may be used as a tracer. To do so, it is necessary to establish a biological background level of ${ }^{129} \mathrm{I}$ and the ${ }^{129} \mathrm{I} /{ }^{127} \mathrm{I}$ ratio in humans. The ${ }^{129} \mathrm{I} /{ }^{127} \mathrm{I}$ ratios in our data ranged from $7.38 \times 10^{-12}$ to $3.97 \times 10^{-10}$ with median of $1.29 \times 10^{-10}$. These values fall within the present background level in the (Zhang and Hou 2013) study. Our ratios are considerably lower than those measured by Hou et al., 2003, where the median ratio in human urine was $1.87 \times 10^{-8}$. However, only 3 samples of urine were measured in (Hou et al. 2003) and cannot be used to establish a robust background level. Nevertheless, as the three urine samples were obtained from three males in Zealand, Denmark it highly probable that the source of these urine samples is responsible for the higher values of ${ }^{129} \mathrm{I} /{ }^{127} \mathrm{I}$. In Denmark, the ${ }^{129} \mathrm{I}$ concentration is heavily influenced by nearby contributors of ${ }^{129} \mathrm{I}$, such as discharges from the nuclear fuel reprocessing plants at Sellafield and La Hague with a small contribution in the past from Chernobyl. Also, the concentration of ${ }^{129} \mathrm{I}$ in this area is influenced by a secondary emission of ${ }^{129}$ I from surface seawater in the North Sea (Kadowaki et al. 2018). In comparison, the source of human urine samples in this study was from Ottawa, Canada, where is the emission of ${ }^{129} \mathrm{I}$ is much lower. The primary source of ${ }^{129} \mathrm{I}$ in North America today is wet deposition of ${ }^{129} \mathrm{I}$ from long-range atmospheric transport of ${ }^{129}$ I from European fuel reprocessing, recycled ${ }^{129}$ I from 
weapons testing and ocean volatilization (Wetherbee et al. 2012). It is well known that the atmospheric deposition of ${ }^{129} \mathrm{I}$ in North America is much lower than western Europe, and the ${ }^{129} \mathrm{I}$ concentration in North American rivers and rain reflects this (Santschi and Schwehr 2004; Sheppard and Herod 2012). ${ }^{129}$ I has been investigated in several sites in Ontario and around Ottawa. For instance, the ${ }^{129} \mathrm{I}$ concentration was measured in Sturgeon Falls groundwater, snow, soil, and grass. The ${ }^{129} \mathrm{I}$ concentration ranges from $1.5 \times 10^{5}$ atoms $/ \mathrm{L}$ to $1.9 \times 10^{6}$ atoms $/ \mathrm{L}$ in groundwater, $8.5 \times 10^{7}$ atoms $/ \mathrm{L}$ in snow, $4.3 \times 10^{8}$ atoms $/ \mathrm{g}$ in the soil, and $7.5 \times 10^{7}$ atoms $/ \mathrm{g}$ in grass (Renaud et al. 2005). These ${ }^{129} \mathrm{I}$ concentrations are very similar to the data presented in this paper. This convergence in concentrations between the Ontario environment and urine of people who live in Ontario supports the earlier assumption that the higher values of ${ }^{129} \mathrm{I} /{ }^{127} I$ of urine in (Hou et al. 2003) compared to this study is related to the location and suggests that there is a strong influence on place of residence to the concentration of ${ }^{129} \mathrm{I}$ and the ${ }^{129} \mathrm{I} /{ }^{127} \mathrm{I}$ ratio in people.

\section{Conclusions}

Iodine-129 was successfully extracted from human urine by using hydrogen peroxide as a digestive solvent and treated by autoclave. Autoclave was chosen because it is available in most labs and provides the same outcome of thermal breakdown as microwave (Taylor et al. 1996) with low cost. Inorganic ${ }^{125}$ I was successfully used as a quantitative recovery tracer. Our extraction technique determined the optimal conditions needed to extract ${ }^{129}$ I from urine efficiently. It was found that a smaller volume of acid $(0.2 \mathrm{ml} \mathrm{HCl})$ after autoclave digestion is the optimal volume for iodine recovery to avoid loss by volatilization. After setting the optimal conditions for extracting ${ }^{129} \mathrm{I}$ from urine, 25 urine samples were analyzed, and the results were compared to three urine samples from Denmark (Hou et al. 2003). It was found that urine from people in the Ottawa area had a mean ${ }^{129} \mathrm{I} /{ }^{127} \mathrm{I}$ ratio of $1.36 \times 10^{-10}$ whereas the Danish samples 
had a mean ratio of $1.87 \times 10^{-8}$. This difference is due to the much closer proximity of Denmark to sources of ${ }^{129} \mathrm{I}$ emission, such as nuclear fuel reprocessing facilities and shows the important influence of place of residence on 129I concentrations and the ${ }^{129} \mathrm{I} /{ }^{127} \mathrm{I}$ ratio in people. The concentration of ${ }^{127} \mathrm{I}$ and ${ }^{129} \mathrm{I}$ in Ottawa urine samples were significantly correlated. This is evidence that both iodine isotopes share a common source of intake into the human body. The ability to measure ${ }^{129} \mathrm{I}$ in human urine expands the application of ${ }^{129} \mathrm{I}$ in the biomedical field and its use as a tracer. This new and reliable method will enable the investigation of the sources of iodine in the human diet and their relative importance for iodine sufficiency. Also, this procedure

can help to evaluate ${ }^{129}$ I exposure directly in the people whom live near or downstream from a contaminated area such as nuclear reprocessing plants.

\section{Conflict of Interests}

The authors declare that they have no conflict of interest.

\section{Human Rights:}

All procedures followed were in accordance with the ethical standards of the Office of Research Ethics and Integrity committee in University of Ottawa, Canada. Informed consent was obtained from all participants for being included in the study.

\section{Animal Rights:}

This article does not contain any studies with animal subjects performed by the any of the authors.

\section{Acknowledgment}


The authors thank their colleagues at Andre E. Lalonde AMS Laboratory for their collaboration and assistance. Fahad AL Otaibi thanks the college of Public Health in University of Hail for their administrative support, especially the Dean, Professor. Fahad AL Qahtani.

\section{Reference:}

Benoist, Bruno de, Maria Andersson, Ines Egli, Bahi Takkouche, and Henrietta Allen. 2004. “lodine Status Worldwide." WHO Global Database on lodine Deficiency. Geneca: World Health Organization, 112.

Brunzel, Nancy. 2013. Fundementals of Urine and Body Fluid Analysis. Elsevier. Elsevier.

Crowther, Joan. 1978. "Autoclave Digestion Procedure for the Determination of Total Iron Content of Waters." Analytical Chemistry 50 (4): 658-59. https://doi.org/10.1021/ac50026a032.

Falls, Sturgeon, By Robert Renaud, I D Clark, T G Kotzer, G M Milton, and D J Bottomley. 2005. “The Mobility of Anthropogenic 129 I in a Shallow Sand Aquifer" 371: 363-71.

FDA, Food and Drug Administration. 2018. "Bioanalytical Method Validation Guidance." Food and Drug Administration 1043 (May): 25.

Fehn, Udo. 1999. "Sources and Reservoirs of Anthropogenic lodine-129 in Western New York." Geochimica et Cosmochimica Acta 63 (13-14): 1927-38. https://doi.org/10.1016/S00167037(99)00133-7.

Fields, Cheryl, and Jonathan Borak. 2009. "Iodine Deficiency in Vegetarian and Vegan Diets: EvidenceBased Review of the World's Literature on lodine Content in Vegetarian Diets." Comprehensive Handbook of lodine, 521-31. https://doi.org/10.1016/B978-0-12-374135-6.00054-6. 
Francisco, B. B.A., R. Bergl, X. L. Zhao, R. J. Cornett, and W. E. Kieser. 2020. “Comparison of Two Methods to Determine 129 I in Charcoal Cartridge Samples by AMS." Nuclear Instruments and Methods in Physics Research, Section B: Beam Interactions with Materials and Atoms 466 (February 2018): 4751. https://doi.org/10.1016/j.nimb.2019.12.015.

García-Toraño, Eduardo, Timotheos Altzitzoglou, Pavel Auerbach, Marie Martine Bé, Christophe Bobin, Philippe Cassette, Frédéric Chartier, et al. 2018. "The Half-Life of 129I." Applied Radiation and Isotopes 140 (June): 157-62. https://doi.org/10.1016/j.apradiso.2018.06.007.

Gómez-Guzmán, J. M., S. M. Enamorado-Báez, A. R. Pinto-Gómez, and J. M. Abril-Hernández. 2011. “Microwave-Based Digestion Method for Extraction Of127I And129I from Solid Material for Measurements by AMS and ICP-MS." International Journal of Mass Spectrometry 303 (2-3): 103-8. https://doi.org/10.1016/j.ijms.2011.01.006.

Gunnarsdottir, Ingibjörg, and Lisbeth Dahl. 2012. “lodine Intake in Human Nutrition: A Systematic Literature Review." Food \& Nutrition Research 56 (1): 19731. https://doi.org/10.3402/fnr.v56i0.19731.

Haldimann, M., A. Eastgate, and B. Zimmerli. 2000. "Improved Measurement of lodine in Food Samples Using Inductively Coupled Plasma Isotope Dilution Mass Spectrometry." Analyst 125 (11): 1977-82. https://doi.org/10.1039/b005879n.

Herod, Matthew N., R. Jack Cornett, Ian D. Clark, W. E. Kieser, and Gilles St Jean. 2014. "Extraction of 129 I and 127I via Combustion from Organic Rich Samples Using $125 \mathrm{I}$ as a Quantitative Tracer." Journal of Environmental Radioactivity 138: 323-30. https://doi.org/10.1016/j.jenvrad.2014.02.005.

Herod, Matthew N., Tianjiao Li, André Pellerin, William E. Kieser, and lan D. Clark. 2016. "The Seasonal 
Fluctuations and Accumulation of lodine-129 in Relation to the Hydrogeochemistry of the Wolf Creek Research Basin, a Discontinuous Permafrost Watershed." Science of the Total Environment 569-570: 1212-23. https://doi.org/10.1016/j.scitotenv.2016.06.196.

Horrocks, Donald L., and Paul R. Klein. 1974. "Theoretical Considerations for Standardization of $125 \mathrm{l}$ by the Coincidence Method." Nuclear Instruments and Methods in Physics Research 124 (3): 585-89.

Hou, Xiaolin, A. F. Malencheko, J. Kucera, H. Dahlgaard, and S. P. Nielsen. 2003. “lodine-129 in Thyroid and Urine in Ukraine and Denmark." Science of the Total Environment 302 (1-3): 63-73. https://doi.org/10.1016/S0048-9697(02)00321-2.

Jabbar, Tania, Gabriele Wallner, and Peter Steier. 2013. "A Review on 129l Analysis in Air." Journal of Environmental Radioactivity 126: 45-54. https://doi.org/10.1016/j.jenvrad.2013.07.013.

Jeffries, Dean S., F. P. Dieken, and D. E. Jones. 1979. "Performance of the Autoclave Digestion Method for Total Phosphorus Analysis." Water Research 13 (3): 275-79. https://doi.org/10.1016/00431354(79)90206-9.

Kadowaki, Masanao, Genki Katata, Hiroaki Terada, Takashi Suzuki, Hidenao Hasegawa, Naofumi Akata, and Hideki Kakiuchi. 2018. "Impacts of Anthropogenic Source from the Nuclear Fuel Reprocessing Plants on Global Atmospheric lodine-129 Cycle: A Model Analysis." Atmospheric Environment 184 (January): 278-91. https://doi.org/10.1016/j.atmosenv.2018.04.044.

Mannar, Venkatesh M.G., and John T. Dunn. 1995. Salt lodization for the Elimination of lodine Deficiency.

May, Sandra L., Warwick A. May, Pierre P. Bourdoux, Sam Pino, Kevin M. Sullivan, and Glen F. Maberly. 1997. "Validation of a Simple, Manual Urinary lodine Method for Estimating the Prevalence of lodine-Deficiency Disorders, and Interlaboratory Comparison with Other Methods." American 
Journal of Clinical Nutrition 65 (5): 1441-45. https://doi.org/10.1093/ajcn/65.5.1441.

Muramatsu, Yasuyuki, Yukari Takada, Hiroyuki Matsuzaki, and Satoshi Yoshida. 2008. "AMS Analysis of 129 I in Japanese Soil Samples Collected from Background Areas Far from Nuclear Facilities." Quaternary Geochronology 3 (3): 291-97. https://doi.org/10.1016/j.quageo.2007.08.002.

Navarrete-López, M., M. P. Jonathan, P. F. Rodríguez-Espinosa, and J. A. Salgado-Galeana. 2012.

"Autoclave Decomposition Method for Metals in Soils and Sediments." Environmental Monitoring and Assessment 184 (4): 2285-93. https://doi.org/10.1007/s10661-011-2117-4.

Pandav, C S, K Yadav, R Srivastava, R Pandav, and M G Karmarkar. 2013. “Iodine Deficiency Disorders (IDD) Control in India." Indian Journal of Medical Research 138 (SEP): 418-33. http://www.scopus.com/inward/record.url?eid=2-s2.084885073178\&partnerID=40\&md5=ad167a344b5401bf05ac4baa3538fe24.

Pino, Sam, Shih Lieh Fang, and Lewis E. Braverman. 1996. “Ammonium Persulfate: A Safe Alternative Oxidizing Reagent for Measuring Urinary lodine." Clinical Chemistry 42 (2): 239-43. https://doi.org/10.1093/clinchem/42.2.239.

Raisbeck, G. M., and F. Yiou. 1999. "129I in the Oceans: Origins and Applications." Science of the Total Environment 237-238: 31-41. https://doi.org/10.1016/S0048-9697(99)00122-9.

Santschi, Peter H, and Kathleen A Schwehr. 2004. "I y 127 I as a New Environmental Tracer or Geochronometer for Biogeochemical or Hydrodynamic Processes in the Hydrosphere and Geosphere : The Central Role of Organo-lodine" 321: 257-71. https://doi.org/10.1016/j.scitotenv.2003.09.003.

Sheppard, S.C., and M. Herod. 2012. "Variation in Background Concentrations and Specific Activities of $36 \mathrm{Cl}, 129 \mathrm{I}$ and U/Th-Series Radionuclides in Surface Waters." Journal of Environmental 
Radioactivity 106 (April): 27-34. https://doi.org/10.1016/j.jenvrad.2011.10.015.

Sunny, Faby, R. N. Nair, Manish Chopra, and V. D. Puranik. 2014. "Global Environmental Transfer of 129I." Annals of Nuclear Energy 65: 320-24. https://doi.org/10.1016/j.anucene.2013.11.036.

Taylor, Clive R., Shan Rong Shi, Chen Chen, Lillian Young, Christina Yang, and Richard J. Cote. 1996. “Comparative Study of Antigen Retrieval Heating Methods: Microwave, Microwave and Pressure Cooker, Autoclave, and Steamer." Biotechnic and Histochemistry 71 (5): 263-70. https://doi.org/10.3109/10520299609117171.

Wetherbee, Gregory A., David A. Gay, Timothy M. Debey, Christopher M.B. Lehmann, and Mark A. Nilles. 2012. "Wet Deposition of Fission-Product Isotopes to North America from the Fukushima Dai-Ichi Incident, March 2011." Environmental Science and Technology 46 (5): 2574-82. https://doi.org/10.1021/es203217u.

Wu, Tingting, and James D. Englehardt. 2012. “A New Method for Removal of Hydrogen Peroxide Interference in the Analysis of Chemical Oxygen Demand." Environmental Science and Technology 46 (4): 2291-98. https://doi.org/10.1021/es204250k.

Zhang, Luyuan Y., and Xiaolin L. Hou. 2013. "Speciation Analysis of 129 I and Its Applications in Environmental Research." Radiochimica Acta 540 (July): 130729000243004. https://doi.org/10.1524/ract.2013.2077.

Zhao, X., W.E. Kieser, H. Tomaru, Z. Lu, and U. Fehn. 2009. “Comparison of Three Chemical Extraction Methods for I-129 Determinations." Nuclear Instruments and Methods in Physics Research Section B: Beam Interactions with Materials and Atoms 268 (7-8): 952-55. https://doi.org/10.1016/j.nimb.2009.10.072.

Zimmermann, Michael B. 2008. "Methods to Assess Iron and lodine Status British Journal of Nutrition," 
2-9. https://doi.org/10.1017/S000711450800679X. 